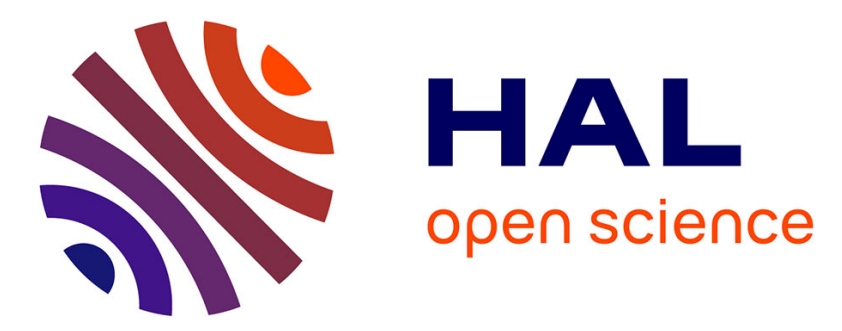

\title{
Comment je fais... une suspension utérovaginale par sacrospinofixation antérieure bilatérale prothétique par voie vaginale
}

\author{
G. Lamblin, E. Cerruto, P. Chabert, K. Lebail Carval, E. Nohuz, G. Chene
}

\section{To cite this version:}

G. Lamblin, E. Cerruto, P. Chabert, K. Lebail Carval, E. Nohuz, et al.. Comment je fais... une suspension utérovaginale par sacrospinofixation antérieure bilatérale prothétique par voie vaginale. Gynécologie Obstétrique Fertilité \& Sénologie, 2019, 47, pp.535 - 539. 10.1016/j.gofs.2019.04.006 . hal-03486585

\section{HAL Id: hal-03486585 \\ https://hal.science/hal-03486585}

Submitted on 20 Dec 2021

HAL is a multi-disciplinary open access archive for the deposit and dissemination of scientific research documents, whether they are published or not. The documents may come from teaching and research institutions in France or abroad, or from public or private research centers.
L'archive ouverte pluridisciplinaire HAL, est destinée au dépôt et à la diffusion de documents scientifiques de niveau recherche, publiés ou non, émanant des établissements d'enseignement et de recherche français ou étrangers, des laboratoires publics ou privés.

\section{(ㅇ)(1) $\$$}

Distributed under a Creative Commons Attribution - NonCommerciall 4.0 International 


\title{
Comment je fais ... une suspension utéro-vaginale par sacrospinofixation antérieure bilatérale prothétique par voie vaginale
}

\section{How I do ... Utero-vaginal suspension using a bilateral vaginal anterior sacrospinous fixation with mesh}

\author{
G. Lamblin ${ }^{\mathrm{a}, \mathrm{b}}$, E.Cerruto ${ }^{\mathrm{a}}$, P. Chabert ${ }^{\mathrm{a}}$, K. Lebail Carval ${ }^{\mathrm{a}}$, E.Nohuz $^{\mathrm{a}}$, G. Chene ${ }^{\mathrm{a}, \mathrm{b}}$ \\ ${ }^{a}$ Service de Chirurgie Gynécologique, Hôpital Femme Mère Enfant, Bron, France \\ ${ }^{\mathrm{b}}$ Université Claude Bernard Lyon 1
}

Mots clés : prolapsus uro-génital ; chirurgie vaginale; suspension apicale, sacrospinofixation antérieure

Key words : pelvic organ prolapse ; vaginal surgery; apical suspension, anterior sacrospinous fixation

\section{Introduction}

La technique de référence du traitement du prolapsus utérin par voie vaginale est la sacrospinofixation vaginale ou intervention de Richter décrite en 1968 [1]. Cette technique était décrite à l'origine comme un traitement des prolapsus post-hystérectomies par voie basse en abordant la fosse para rectale par voie postérieure. Elle consiste à fixer le dôme vaginal au ligament sacro-spinal et amener la colonne vaginale au-dessus du plancher lévatorien selon l'axe S3. Les derniers travaux de Delancey plaident en faveur de la nécéssité d'une réparation des niveaux I (utérus/dôme vaginal) et niveau II (1/3 moyen du vagin) pour le traitement d'une hystéroptose de stade 3 avec cystocèle apicale [2]. La sacrospinofixation modifiée par voie antérieure a été décrite pour la première fois par Winkler et al. [3,4]. Cette technique peut être utilisée pour les patientes éligibles à une cure de prolapsus prothétique par voie vaginale, c'est-àdire présentant une cystocèle récidivée après échec d'une technique de réparation autologue ou de 
stade POP-Q $\geq 3$ avec des facteurs de risques de récidive. De récentes études ont évalué l'efficacité anatomique avec un taux de succès de $93 \%$ au niveau des compartiments antérieur et apical pour un suivi médian de 23 mois et un taux de satisfaction des patientes à 95\% [4,5]. De plus, les prothèses de 3ème génération sont actuellement plus petite et de faible grammage (entre 19 et $25 \mathrm{~g} / \mathrm{m}^{2}$ ) permettant une meilleure tolérance. Nous présentons une technique mini invasive de suspension utéro-vaginale prothétique par une approche antérieure bilatérale du ligament sacrospinal dont l'apprentissage doit actuellement être enseigné dans des centres experts selon les données récentes de l'ANSM.

\section{Description de la technique chirurgicale}

L'intervention se déroule sous anesthésie générale ou loco régionale. La patiente est installée en position gynécologique. Après aseptie à la polyvidone iodée et antibioprophylaxie ( $2 \mathrm{~g}$ amoxicilline-acide clavulanique $\left(\right.$ Augmentin $^{\circledR}$ ) par voie intra-veineuse en absence d'allergie et champage stérile, une sonde urinaire à demeure est mise en place (fig 1.a). L'hystérectomie ou l'amputation cervicale ne sont pas nécessaires, et doivent être envisagées en présence d'une élongation cervicale ou d'une pathologie utérine associé (adénomyose, fibromes). L'intervention débute par une infiltration de la paroi vaginale antérieure avec 30 cc de xylocaïne adrénaliné $1 \%$ dilué pour moitié avec du sérum physiologique permettant de faciliter la dissection (fig 1.b). En cas de contre-indication à l'utilisation de l'adrénaline, on a recours au sérum physiologique seul afin de mettre à profit l'hydro dissection (fig 1.b). L'infiltration se fait le long de l'incision vaginale, puis en direction de l'épine sciatique, de manière à infiltrer la fosse paravésicale. La colpotomie antérieure se fait longitudinalement, débutant à $3 \mathrm{~cm}$ du méat urétral et se poursuivant jusqu'à $2 \mathrm{~cm}$ environ du col utérin (fig 1.c). L'incision doit être profonde, intéressant la totalité de l'épaisseur de la paroi vaginale antérieure et le fascia inter-vésicovaginal (fig 1.d). Point important, la dissection est réalisée au bistouri froid, sous le fascia inter-vésicovaginal qui doit rester au contact de la paroi vaginale antérieure (fig 1.d). Elle se fait en direction de la branche ischiopubienne permettant d'ouvrir la fosse paravésicale (fig 1.e,1.f), initiée aux ciseaux puis la progression est assurée au doigt, en longeant de haut en bas l'arcus tendineus du fascia pelvien (ATFP) par un mouvement de balayage jusqu'au relief de l'épine sciatique (fig 1.g). Il est parfois nécessaire d'ouvrir aux ciseaux l'aponévrose pelvienne profonde afin d'accéder au ligament sacrospinal (fig 1.h). L'approche antérieure du ligament sacro-spinal nécessite sa dissection à partir de l'épine sciatique sur au moins $2 \mathrm{~cm}$. La même dissection est effectuée du côté controlatéral. Il est 
nécessaire de dégager la face antérieure du col utérin jusqu'à 2 à $3 \mathrm{~cm}$ de l'incision initiale afin de pouvoir fixer solidement la prothèse à l'isthme utérin en fin d'intervention (fig 1.i). On place ensuite 2 fils non résorbables (type Prolène ${ }^{\circledR} 2 / 0$ ) sur les bords latéraux du col utérin à au moins 2 $\mathrm{cm}$ de l'incision vaginale médiane afin que la colporraphie antérieure puisse être à distance des implants prothétiques (fig 1.i).

L'opérateur et les aides procèdent au changement de gants avant l'étape de pose de la prothèse. Le passage du bras de la prothèse dans le ligament sacro-spinal nécessite l'utilisation d'un dispositif transfixant le ligament sacro-spinal de type Capio ${ }^{\circledR}$ (Boston scientific), Digitex ${ }^{\circledR}$ (Coloplast), Istich $^{\circledR}$ (AMI), Anchorsure system ${ }^{\circledR}$ (Neomedic international) (fig 2.a). Ces dispositifs de fixation permettent de faire passer l'aiguille à travers le ligament sacro-spinal (fig 2.b, 2.c). Nous décrirons le procédé faisant appel au dispositif Capio ${ }^{\circledR}$. L'index de l'opérateur expose l'épine sciatique et le ligament sacro-spinal, ce qui permet l'introduction du dispositif parallèlement au doigt afin de sécuriser le geste et réduire le risque de plaie, notamment vésicale (fig 3.a). L'instrument doit alors cheminer dans la fosse paravésicale jusqu'à arriver au contact de l'épine sciatique (fig 3.b). Il est important qu'aucune tension ne soit exercée sur la prothèse au moment de l'action du piston du dispositif qui permet à l'aiguille de traverser le ligament sacro- spinal. Le doigt va ensuite aider le positionnement de l'instrument sur le ligament sacro-spinal à $2 \mathrm{~cm}$ de l'épine sciatique afin de limiter les risques de blessure notamment du nerf pudendal, avant d'appuyer sur le piston. L'index de l'opérateur stabilise la tête du dispositif contre le ligament sacro-spinal à l'aide d'une légère pression sur sa partie crantée. Une fois le ligament traversé, l'ensemble du dispositif est alors retiré et le fil est mis en attente sur une pince Kocher. L'index vérifie le bon positionnement sur le ligament sacro-spinal à $2 \mathrm{~cm}$ de l'épine sciatique (fig 3.c, 3.d). Les bras de la prothèse doivent être non «twistés » de manière à éviter une accroche dans le ligament sacro-spinal (fig 3.d). La prothèse est étalée sous la vessie à l'aide d'une pince atraumatique afin de limiter le contact avec les gants et réduire les risques de contamination (fig 3.e).

Un premier temps de réglage va permettre le positionnement de la gaine recouvrant le bras de la prothèse dans le ligament lui-même, le doigt de l'opérateur pouvant à nouveau être introduit de façon à s'assurer de l'absence de croisement entre ladite gaine et le dilatateur (à noter que la gaine reste apparente pour permettre de fixer la prothèse sur le col utérin). Le réglage du bras de la prothèse va être réalisé de façon similaire, en vérifiant l'absence de «twist» du bras, en positionnant l'index contre le ligament afin de s'assurer de la facilité d'introduction de la gaine dans le ligament. La prothèse peut alors être solidarisée au col utérin par les 2 fils mis en attente initialement (fig 3.e). Pour faciliter la fermeture vaginale, nous positionnons le fil de fermeture par 
un fil résorbable $2 / 0$. Le deuxième temps de réglage de la prothèse concerne son emplacement sous le col vésical : la gaine ressort de 4 à $6 \mathrm{~cm}$ environ, ce qui permet de contrôler la symétrie de positionnement de la prothèse (fig 3.f, 3.g).

La prothèse est ensuite fixée sous le col vésical par des fils résorbables permettant un bon étalement sans replis : 2 points latéraux seront placés entre la partie para-médiane de la prothèse et le fascia qui se trouve latéralement par rapport au col vésical (fig 3.g). Le renfort prothétique doit rester relativement lâche à ce stade et sera tendu ultérieurement. Le troisième temps de réglage de la prothèse consiste à tendre les bras pour remonter l'utérus. Une vérification de l'absence de tension dans les culs-de-sac latéraux du vagin est nécessaire. Une fois la tension réglée, le retrait des gaines se fait en sectionnant les deux fils à l'extérieur de la gaine, le doigt contrôlant l'absence d'excès de tension du bras et la main opposée tractant doucement sur la gaine pour ne pas risquer d'en déchirer l'extrémité (la gaine doit être contrôlée au décours de son ablation afin de vérifier l'absence de déchirure). Du côté opposé, les fils qui sortent de la gaine sont également coupés pour désolidariser le bras de la gaine (fig 3.h). Le col utérin est maintenant repositionné, la prothèse est pratiquement horizontale, sans plis, mais sans excès de tension. Une colpectomie n'est pas nécessaire. On ferme ensuite la colpotomie antérieure par un surjet passé de fils monobrin résorbables $2 / 0$ en prenant toute l'épaisseur de la paroi vaginale antérieure (fig 3.i). Après la fermeture complète du vagin, les bras de la prothèse peuvent être encore détendus par un simple positionnement du doigt de l'opérateur dans les culs-de-sac latéraux du vagin. Il est conseillé de pratiquer une cystoscopie en cas de doute sur une plaie vésicale ou une compression urétérale afin de confirmer l'intégrité urétéro-vésicale. L'examen en fin d'intervention montre un col utérin qui est bien positionné, une paroi antérieure horizontale et l'on vérifie une ultime fois par un toucher vaginal l'absence d'excès de tension des bras (fig 4). Un toucher-rectal vérifie l'absence de tension excessive des bras prothétiques. La sonde à demeure est laissée en place pendant $24 \mathrm{~h}$.

\section{Conclusion}

La technique de suspension utéro-vaginale par sacrospinofixation antérieure bilatérale prothétique par voie vaginale permet la correction des 2 niveaux de Delancey. Elle permet la restauration anatomique avec une suspension apicale et une fixation ligamentaire rigide stabilisant l'ensemble de la voûte vaginale antérieure. Notre technique s'inscrit dans la lignée des procédures minimales invasives et s'avère efficace pour la restauration anatomique et fonctionnelle des hystéroptoses de stade 3 avec cystocèle apicale. 
Tous les auteurs ont lu et approuvé la version finale

Conflit d'intérêt: aucun 


\section{Références}

[1] Richter K. The surgical anatomy of the vaginae fixation sacrospinalis vaginalis. A contribution to the surgical treatment of vaginal blind pouch prolapse. Geburtshilfe Frauenheilkd. 1968;28(4):321-7.

[2] DeLancey JO. Anatomic aspects of vaginal eversion after hysterectomy. Am J Obstet Gynecol 1992 Jun;166:1717-24.

[3] Winkler HA, Tomeszko JE, Sand PK. Anterior sacrospinous vaginal vault suspension for prolapse. Obstet Gynecol. 2000 Apr;95(4):612-5.

[4] Goldberg RP, Tomezsko JE, Winkler HA, Koduri S, Culligan PJ, Sand PK. Anterior or posterior sacrospinous vaginal vault suspension: long-term anatomic and functional evaluation. Obstet Gynecol. 2001 Aug;98(2):199-204.

[5] Letouzey V, Ulrich D, Balenbois E, Cornille A, de Tayrac R, Fatton B. Utero-vaginal suspension using bilateral vaginal anterior sacrospinous fixation with mesh: intermediate results of a cohort study. Int Urogynecol J. 2015 Dec;26(12):1803-7. 
Figure 1. Description chirurgicale des différents temps de dissection vaginale, fosses para vésicales et abord antérieur du ligament sacro-spinal.

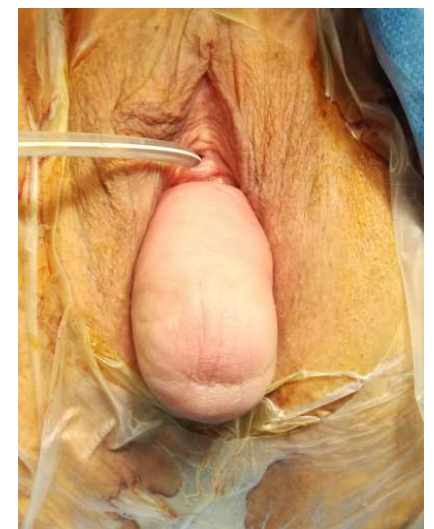

a)

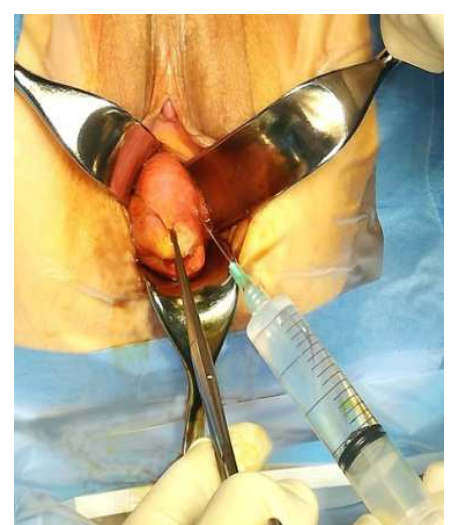

b)

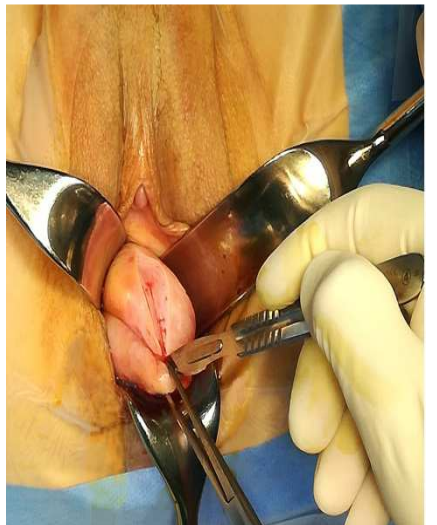

c)

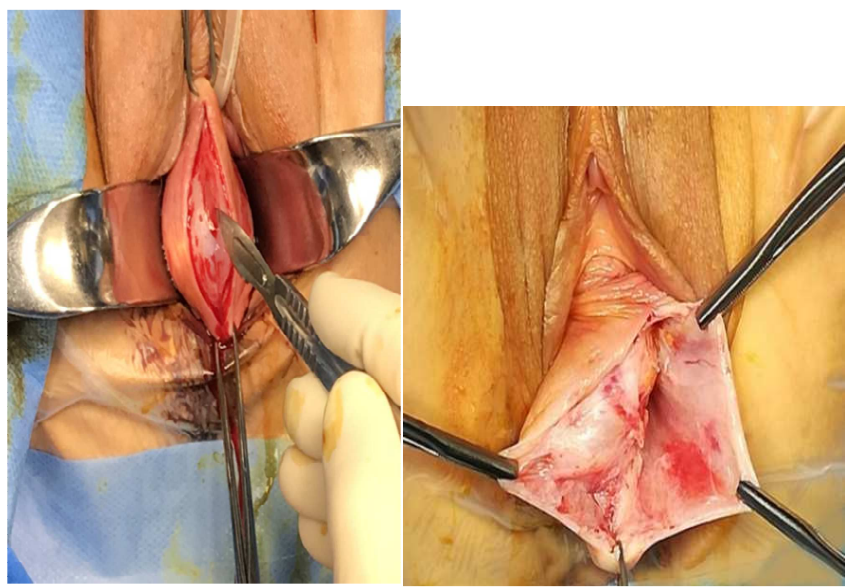

d)

e)

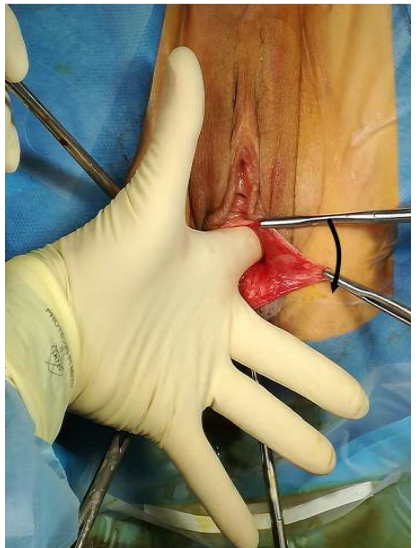

g)

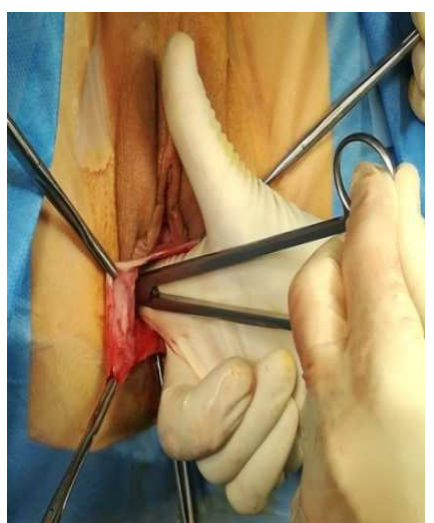

h)

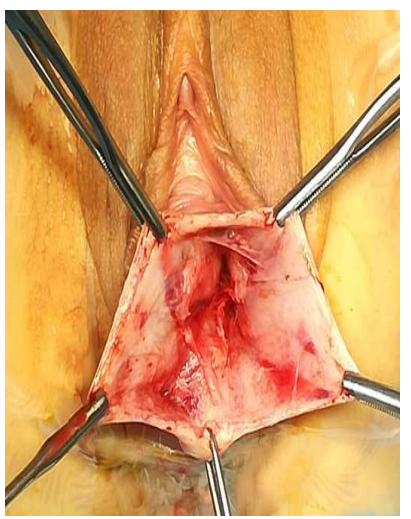

f)

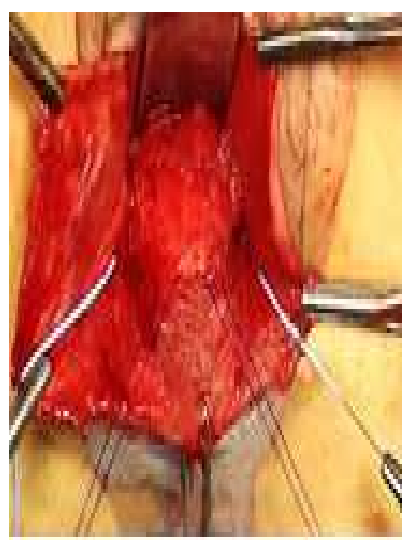

i) 
Figure 2. Différents dispositifs de sacrospino-fixation, Capio ${ }^{\mathrm{TM}}$ (Boston Scientific),

Digitex $^{\mathrm{TM}}$ (Coloplast), I-Stich ${ }^{\mathrm{TM}}$ (AMI), Anchorsure system ${ }^{\mathrm{TM}}$ (Neomedic international)

2.a

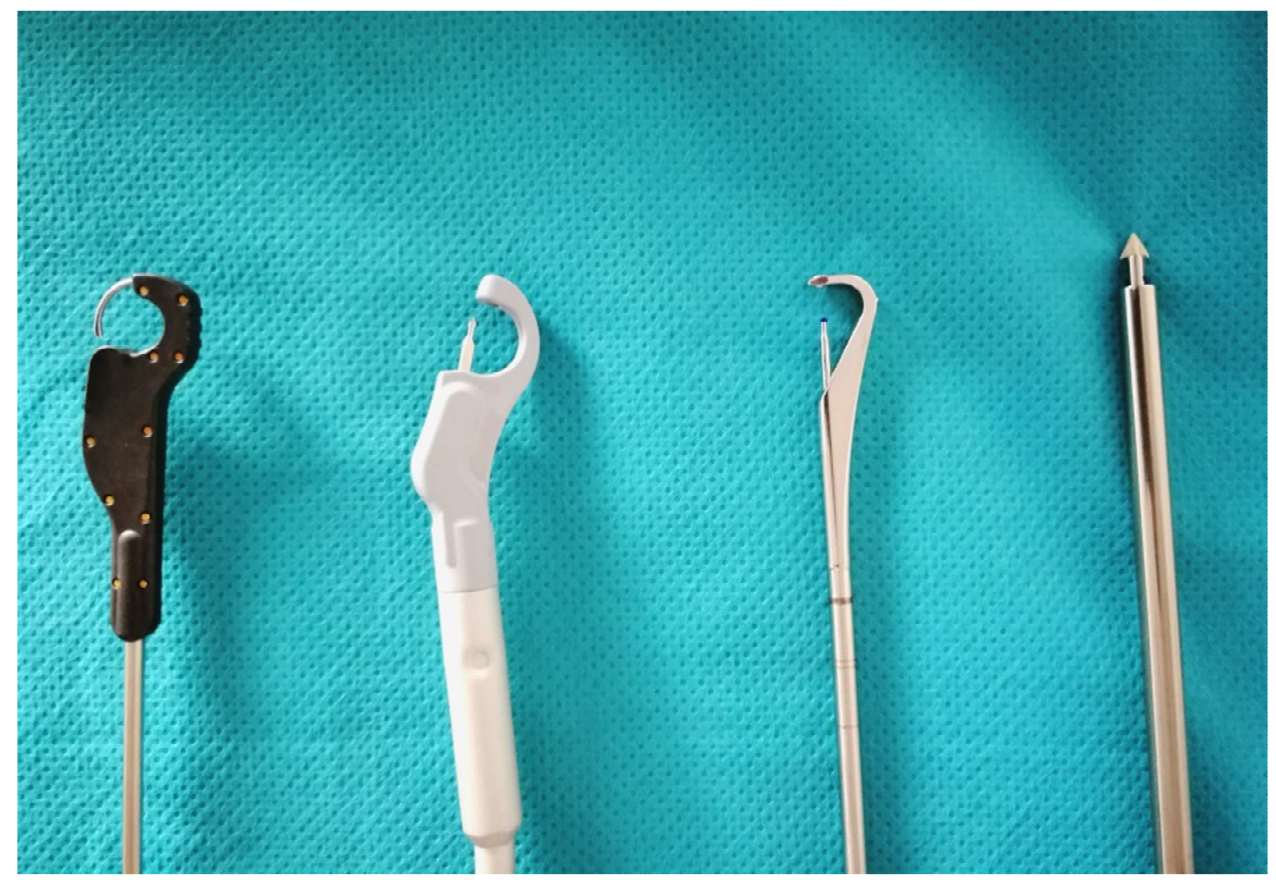

2.b

2.c
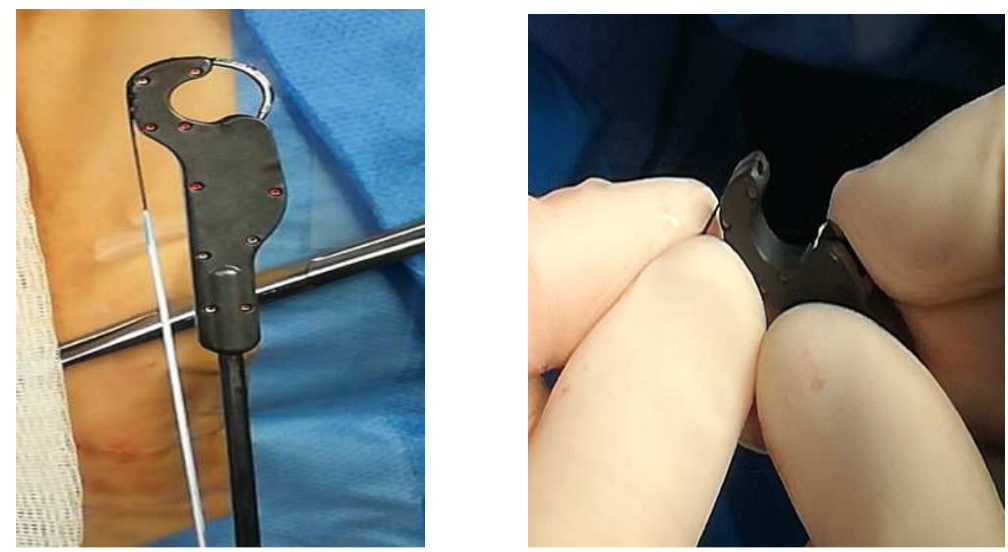
Figure 3. Description chirurgicale des différents temps de fixation au ligament sacro-spinal.

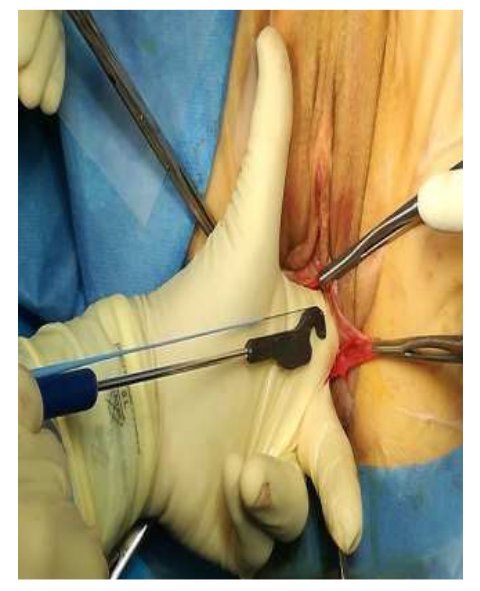

a)

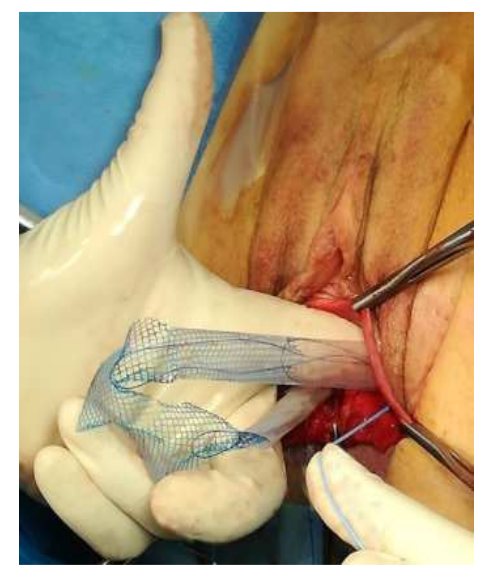

d)

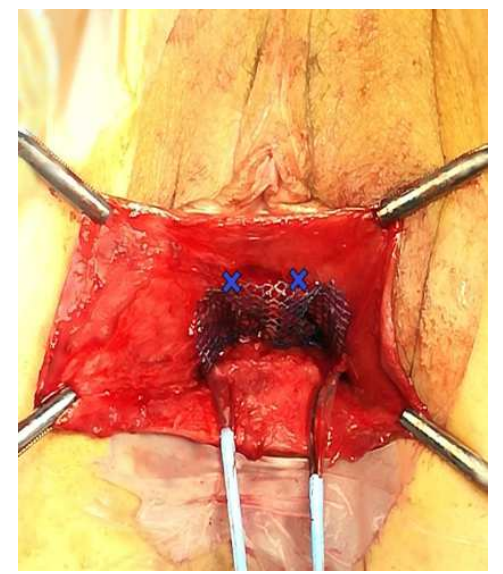

g)

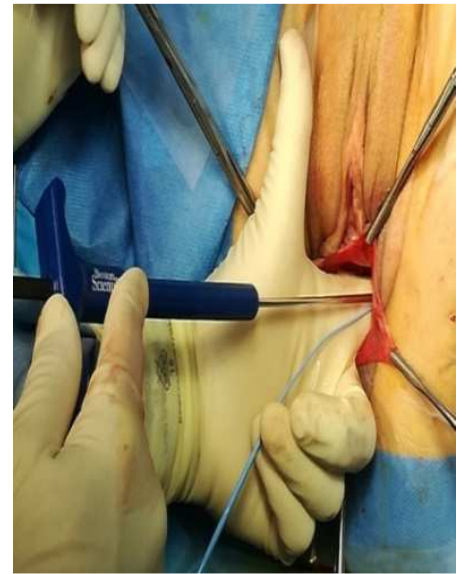

b)

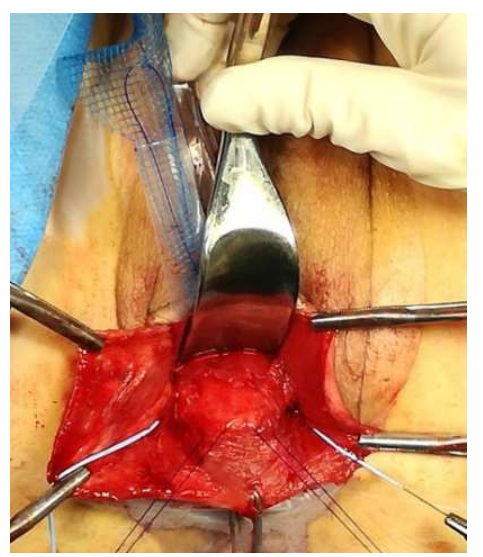

e)

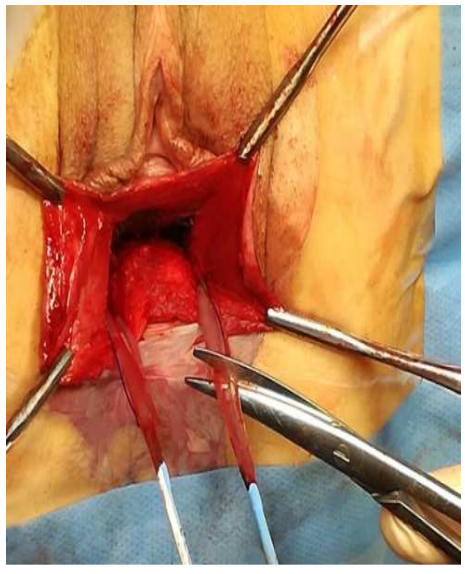

h)

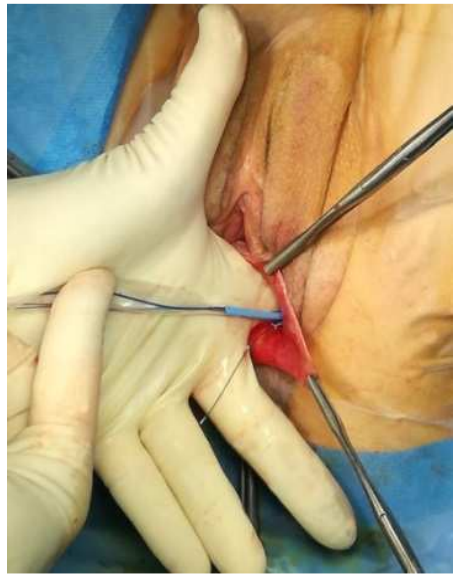

c)

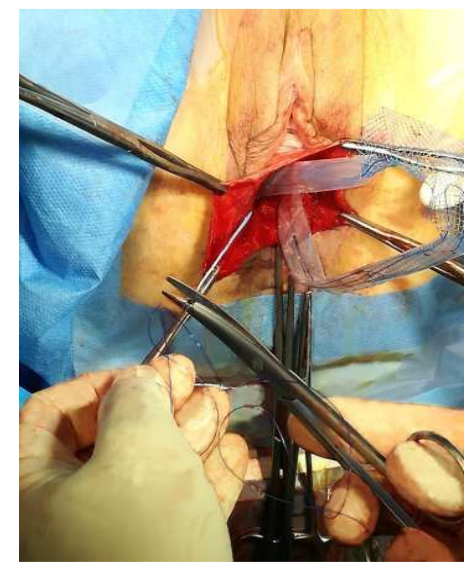

f)

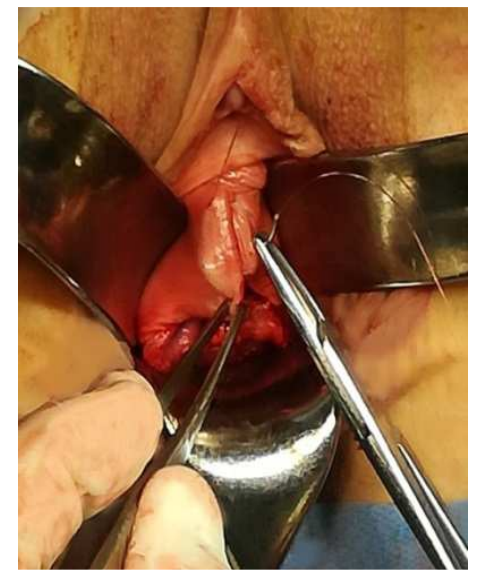

i) 
Figure 4. Résultat anatomique final après correction, point C à -6 (classification POP-Q)

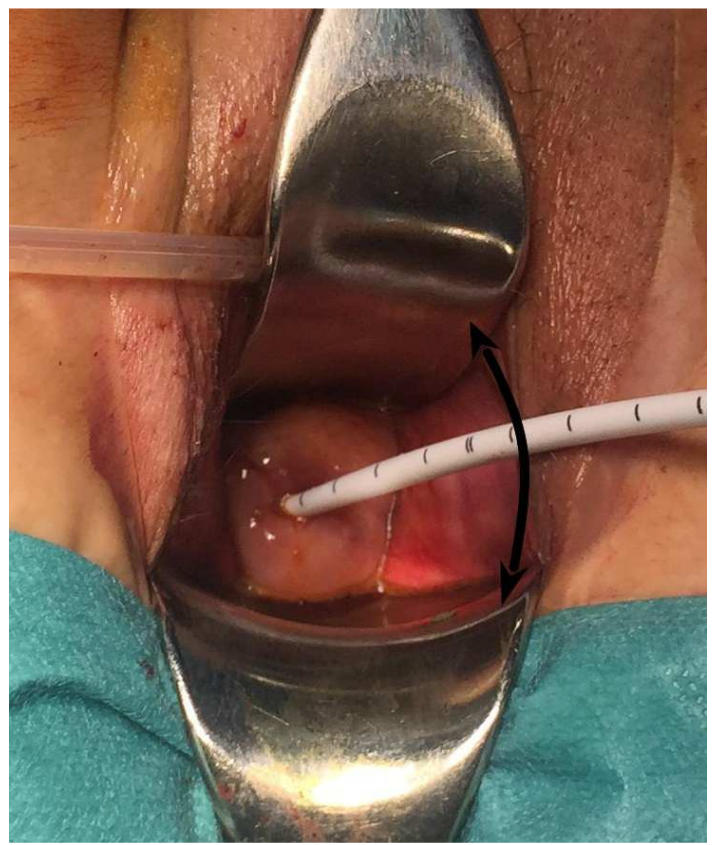

